\title{
Modelagem Geoestatística do Campo de Namorado na Bacia de Campos
}

Felipe Timóteo, Adalberto da Silva - Departamento de Geologia e Geofísica - Universidade Federal Fluminense

Copyright 2016, SBGf - Sociedade Brasileira de Geofísica

Este texto foi preparado para a apresentação no VII Simpósio Brasileiro de Geofísica, Ouro Preto, 25 a 27 de outubro de 2016. Seu conteúdo foi revisado pelo Comitê Técnico do VII SimBGf, mas não necessariamente representa a opinião da SBGf ou de seus associados. É proibida a reprodução total ou parcial deste material para propósitos comerciais sem prévia autorização da SBGf.

\section{Resumo}

A abordagem geoestatística permite a quantificação da variabilidade espacial diretamente dos dados através do variograma, possibilitando assim a criação de modelos de reservatório que simulem, com bastante realismo, a heterogeneidade do sistema. A modelagem geoestatística proposta nesse trabalho foi aplicada ao Campo de Namorado na Bacia de Campos. Foram utilizados os perfis Densidade "Bulk", Porosidade Neutrônica e o parâmetro DRDN para a análise geoestatística através do software SGeMS. A simulação sequencial gaussiana foi realizada para o parâmetro DRDN, que é uma variável contínua que permite a classificação das litofácies, e para a propriedade densidade bulk, utilizada para o cálculo da porosidade total nas regiões onde a fácies reservatório foi classificada pela variável DRDN. A distribuição espacial das litofácies obtida com a simulação sequencial gaussiana forneceu geometrias de reservatório que são coerentes com os sistemas de deposição descritos na literatura. Por fim, os resultados da validação cruzada mostram que os modelos criados a partir da simulação sequencial gaussiana apresentam estabilidade, dessa forma representando bem a variabilidade dos dados e garantindo a heterogeneidade esperada do modelo.

\section{Introdução}

Os métodos geoestatísticos vêm sendo cada vez mais utilizados para a modelagem de reservatórios de petróleo complementando os métodos convencionais. A vantagem dos métodos geoestatísticos sobre os outros métodos é que a abordagem geoestatística é capaz de capturar o comportamento espacial diretamente dos dados através da análise das funções aleatórias, estimando ou simulando, dessa forma, a propriedade de interesse com bastante realismo. Sabendo que a complexidade dos fenômenos geológicos não pode ser adequadamente descrita por modelos físicos determinísticos simplificados, a incerteza espacial inerente aos fenômenos naturais pode ser capturada pelas várias realizações equiprováveis produzidas por uma simulação estocástica. Dessa maneira, a modelagem geoestatística pode fornecer resultados com relativa confiabilidade mesmo com escassez de dados.

A metodologia abordada nesse trabalho foi aplicada ao Campo de Namorado na Bacia de Campos utilizando apenas dados de poços com as respectivas marcações de topo e base do reservatório interpretadas previamente na sísmica e nos perfis de poço. O Campo de Namorado na Bacia de Campos possui uma abundância de estudos e os resultados obtidos nesse trabalho poderão ser comparados com os modelos de reservatórios já propostos por alguns autores.

A Bacia de Campos está localizada na porção sudeste do Brasil, ao longo da costa norte do Estado do Rio de Janeiro. Possui uma área aproximada de 100 mil km², sendo $500 \mathrm{~km}^{2}$ relativos à porção emersa, atingindo lâminas d'água de até $3000 \mathrm{~m}$. Os principais reservatórios de águas profundas da Bacia de Campos; são constituídos por turbiditos e fácies de águas profundas associadas. O Campo de Namorado foi um dos primeiros grandes produtores de petróleo da Petrobrás. Descoberto em 1975, a cerca de $80 \mathrm{~km}$ do litoral e sob uma lâmina d'água de 110 a $250 \mathrm{~m}$, foi o primeiro campo gigante a ser descoberto na plataforma continental brasileira. Sua locação foi baseada em interpretação sísmica de um alto estrutural presente no topo da Formação Macaé (carbonatos de idade albiana). Entretanto, os reservatórios do Campo de Namorado foram encontrados em arenitos turbidíticos, em profundidades entre $2940 \mathrm{~m}$ à $3300 \mathrm{~m}$.

O reservatório foi interpretado como tendo sido formado pela coalescência de canais e lobos depositados sobre uma superfície deposicional irregular. A área onde se encontra o campo de óleo comportava-se como um baixio onde os turbiditos foram trapeados. Como resultado da atividade estrutural relacionada ao movimento de sal no Cretáceo Superior, ocorreu uma inversão de relevo. O reservatório é um domo alongado, parcialmente falhado pelo alto estrutural, indicando um forte controle tectônico associado ao movimento de sal na sedimentação, conferindo ao campo uma estratigrafia complexa. Assim, a acumulação de óleo no Campo de Namorado é controlada pela estratigrafia e estrutura.

Barboza et al. (2005) propõe um modelo deposicional para o Campo de Namorado em que há um empilhamento de leques turbidíticos preenchendo uma depressão estreita e alongada. A deposição apresenta na base uma arquitetura de preenchimento do tipo canalizada e tende à geometria lobada para o topo do intervalo; essa evolução é pontuada por ciclos regressivos e apresenta em termos de evolução uma tendência transgressiva.

\section{Metodologia/ Problema Investigado}

A base de dados utilizada do Campo de Namorado é composta por 37 poços, cada um contendo 11 curvas no formato padrão .LAS, sendo que entre esses poços 14 são verticais e 23 são direcionais. As curvas incluem os dados originais adquiridos e os parâmetros secundários calculados a partir deles. A base de dados também contém uma tabela com a localização em coordenadas UTM para cada poço, incluindo as profundidades Topo e Base do reservatório Namorado obtidos através da sísmica e da análise dos perfis. 


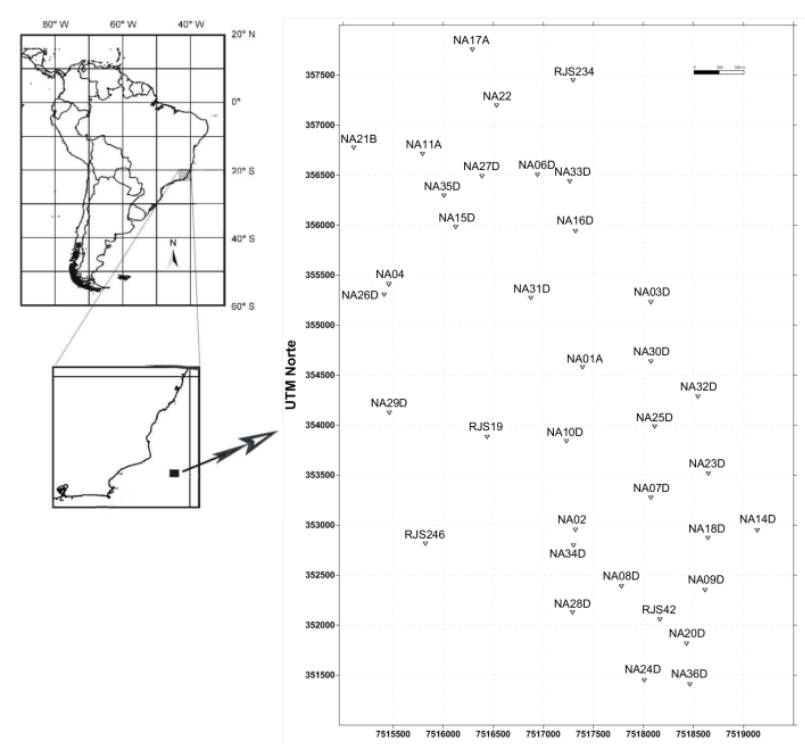

Figura 1- Localização dos poços utilizados no Campo de Namorado

A distribuição dos poços segue uma orientação na direção NW-SE, que é a orientação do paleocanal meandrante presente no Campo de Namorado (BARBOZA, 2005).

Os poços direcionais não possuem informações da trajetória real do poço, somente as coordenadas dos poços com as respectivas profundidades de topo e base do reservatório. Dessa forma, para os poços direcionais, admitindo que a trajetória seja retilínea, foi necessária a transformação dos poços direcionais em poços verticais. A transformação realizada assume que um determinado ponto do perfil permaneça na mesma camada estratigráfica.

Outra transformação realizada nos dados, com o objetivo de garantir uma melhor continuidade lateral, foi a translação vertical ou nivelamento dos poços de maneira que todos possuam a mesma profundidade de base do reservatório, escolhida em $3100 \mathrm{~m}$, que é a profundidade média da base do reservatório. Tal procedimento deve reduzir os erros nas simulações associados às descontinuidades laterais provocadas pelas falhas pósdeposicionais no Campo de Namorado originadas majoritariamente pela movimentação halocinética. Para efeito desse trabalho, entretanto, a restituição estrutural do reservatório não foi realizada após a geração dos grids.

A análise exploratória dos dados foi realizada utilizando o software SGeMS. As variáveis utilizadas nesse trabalho foram Densidade Bulk (RHOB), Porosidade Neutrônica (NPHI), DRDN (o perfil DRDN é uma combinação entre os perfis RHOB e NPHI que fornecem uma relação de fácil visualização entre a presença de arenito, para valores negativos, e folhelhos, para valores positivos).

O procedimento geral para a escolha do melhor modelo variográfico foi realizado da seguinte maneira: primeiro foi admitido que o valor do patamar do variograma experimental é igual à variância $\sigma^{2}$ calculada a partir dos dados, para todas as direções; em seguida foi calculado um variograma omnidirecional (que independe da direção) na menor escala possível para a identificação da contribuição do efeito pepita; por fim, a curva é ajustada aos pontos do variograma experimental para cada direção levando em consideração o número de amostras existentes para cada ponto calculado, consequentemente definindo o alcance.

Através da análise variográfica foi estabelecido, primeiramente, que os variogramas apresentavam anisotropia em todas as direções principais. Entretanto, as simulações utilizando os modelos de variograma com tais anisotropias se mostraram não aceitáveis no plano horizontal $X Y$ pois os resultados apresentavam uma geometria não condizente com os modelos propostos para o Campo de Namorado. Dessa forma os modelos adotados para os variogramas foram isotrópicos para as direções horizontais e apresentando anisotropia com a direção vertical, como era de se esperar, já que a variabilidade na direção vertical é muito maior que nas direções horizontais.

A seguir estão os variogramas omnidirecional, horizontal e vertical para as variáveis analisadas. Os pontos em vermelho representam os valores calculados para 0 variograma a partir dos dados e a linha contínua representa o modelo ajustado aos dados. O variograma omnidirecional foi gerado com o objetivo de visualizar o comportamento geral da variabilidade da variável.

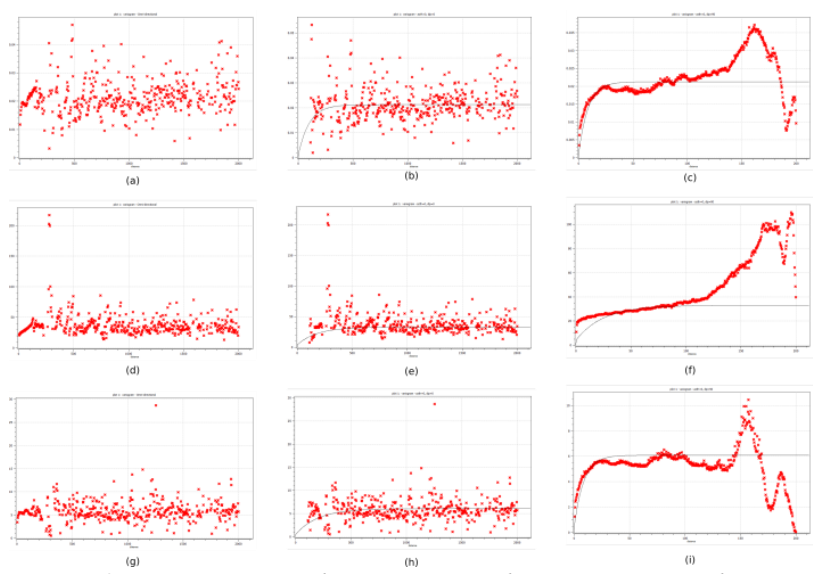

Figura 2-Variogramas de RHOB (a), (b) e (c); NPHI (d), (e) e (f); e DRDN (g), (h) e (i).

A partir dos modelos variográficos estabelecidos para as variáveis RHOB e DRDN foram realizadas as Simulações Sequenciais Gaussiana. O grid tridimensional escolhido para a realização das simulações possui células com dimensão de $20 \mathrm{~m} \times 20 \mathrm{~m}$ para o plano horizontal e $1 \mathrm{~m}$ para a direção vertical possuindo 204 células na direção x, 320 células na direção y e 253 células na direção vertical, dessa forma cobrindo todos os poços disponíveis. 


\section{Resultados}

Foram geradas 10 realizações para as variáveis DRDN e RHOB utilizando a simulação sequencial gaussiana. $O$ número de realizações foi suficiente pois a decisão do número de cenários pode ser avaliada através da flutuação da variância das médias das realizações. Sabese que esse parâmetro apresenta uma oscilação conforme aumentamos os números de realizações até atingir um determinado patamar limite. De forma que o número de cenários ideal seria aquele que garanta que esse patamar tenha sido atingido. Portanto, como mostra a Figura 3 , as 10 realizações feitas são suficientes para mapear a incerteza associada à estimativa da variável analisada.

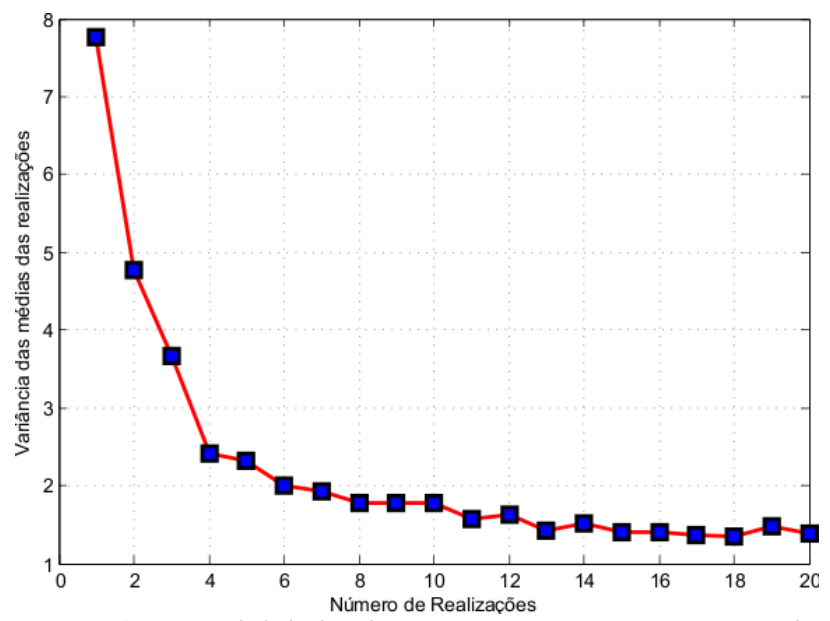

Figura 3- Variabilidade da variância com o aumento do número de realizações em função do número de realizações geradas por $S S G$.

O modelo de reservatório foi obtido calculando a média pontual de cada nó do grid para as 10 realizações equiprováveis da variável DRDN, denominado mapa Etype (figura 4). Como a variável DRDN classifica as litofácies entre rocha reservatório e rocha não reservatório, nos pontos do grid onde foi estabelecida a presença de rocha reservatório foi calculada a porosidade $\varphi_{E}$ através do mapa E-type construído para a variável RHOB, também construído a partir das 10 realizações equiprováveis, utilizando a equação:

$$
\varphi_{E}=\frac{\rho_{m}-R H O B}{\rho_{m}-\rho_{f}} \times 100
$$

onde para o reservatório Namorado a densidade da matriz rocha $\left(\rho_{m}\right)$ é 2.65 e a densidade do fluido no poro $\left(\rho_{f}\right)$ é 1.04. A distribuição espacial das litofácies alcançada com a SSG juntamente com seu respectivo mapa E-type (Figura 5 e 6 ) forneceu geometrias que são coerentes com os sistemas de deposição turbidíticos citados por Souza (1997) e Barboza (2005) capturando a heterogeneidade e complexidade de tais sistemas. Portanto, o método de simulação estocástica é uma excelente ferramenta para a análise da variabilidade espacial de modelos de reservatórios turbidíticos.

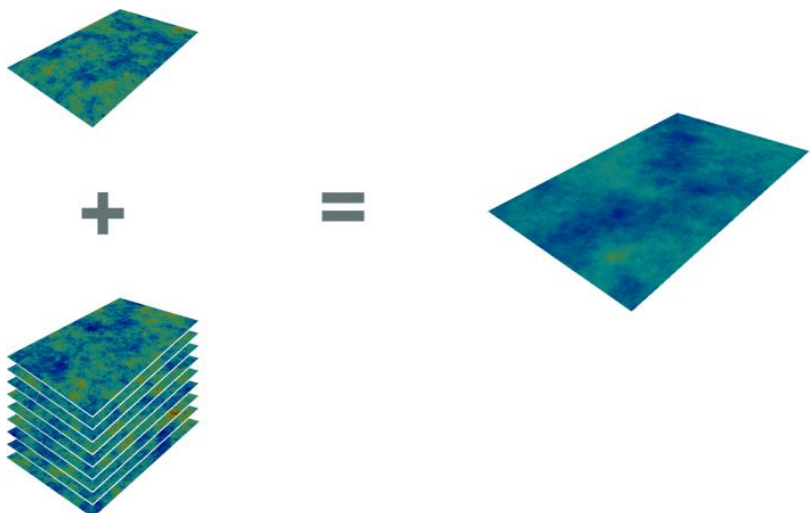

Figura 4 - Representação do empilhamento dos grids para a geração do mapa E-type.

A validação cruzada é um método que permite checar se o modelo criado a partir das técnicas geoestatísticas contém algum tipo de problema, permitindo comparar os valores simulados (ou estimados) com os dados usando apenas as informações disponíveis nos dados.

A ideia básica é estimar o valor da propriedade em localizações onde o valor real é conhecido, descartando temporariamente as amostras de dados nessas posições. O valor nessa mesma localização é então estimado usando as amostras restantes. Uma vez tendo o valor estimado, podemos compará-lo ao verdadeiro valor, inicialmente removido do conjunto de dados, usando um gráfico de dispersão.

Primeiramente, foram removidos dos dados originais $10 \%$ dos poços, realizada a SSG e comparados os valores simulados nas coordenadas dos dados reais, para as variáveis DRDN (Figura 7a) e RHOB (Figura 7e). O mesmo foi realizado removendo $15 \%$ dos poços (Figura $7 \mathrm{~b}$ e Figura $7 \mathrm{f}$ ), $20 \%$ dos poços (Figura $7 \mathrm{c}$ e Figura $7 \mathrm{~g}$ ) e por fim, 30\% dos poços (Figura $7 \mathrm{~d}$ e Figura $7 \mathrm{~h}$ ).
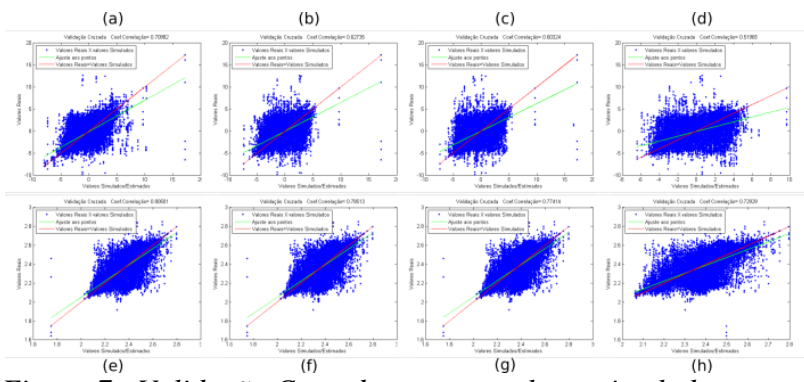

Figura 7 - Validação Cruzada entre os valores simulados para as variáveis DRDN e RHOB

Analisando o coeficiente de correlação de cada validação cruzada, nota-se que mesmo as SSG com $30 \%$ poços a menos, ainda possuem uma boa correlação (acima de 0.51 ) com os dados originais. Portanto, mesmo com a redução de $30 \%$ dos dados, a simulação sequencial gaussiana reproduz resultados estáveis que representam bem a variabilidade dos dados nos modelos alcançados. 


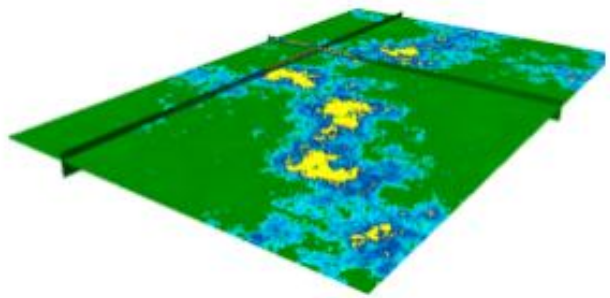

(a)

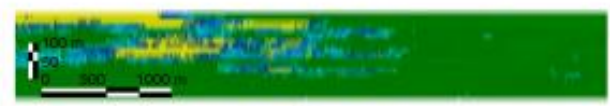

(c)

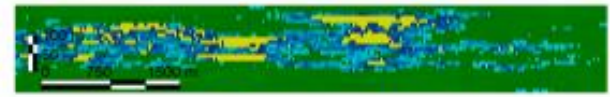

(e)

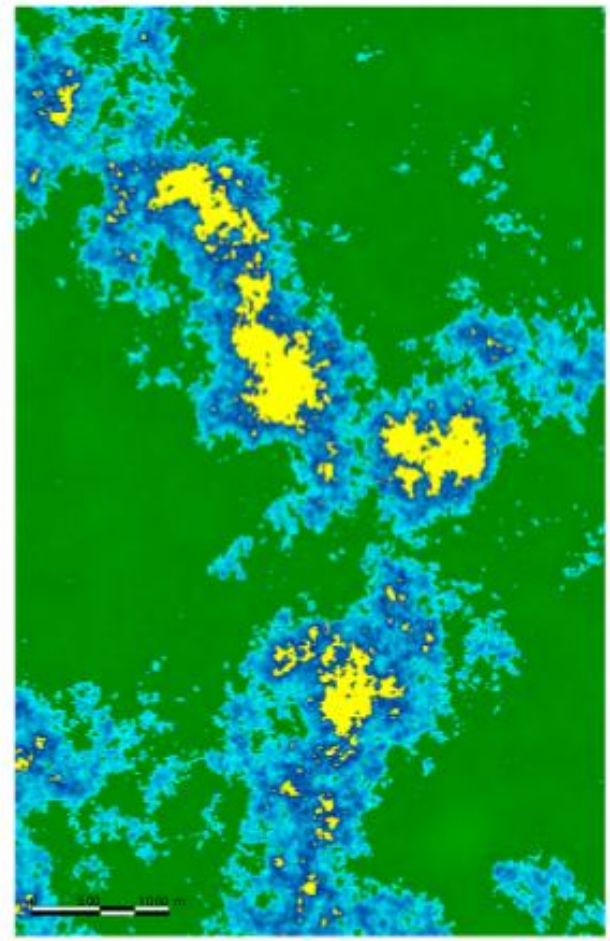

(g)

E NDO FESRTMTORIO I RESERMTORIO IIRESRAMTORO LMPO

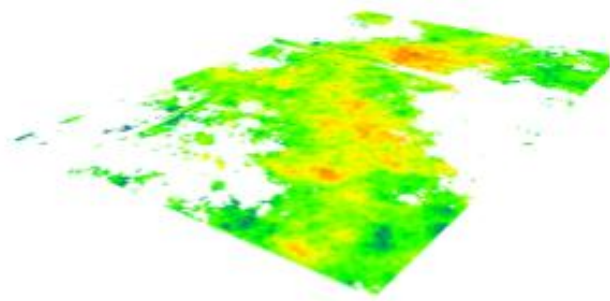

(b)

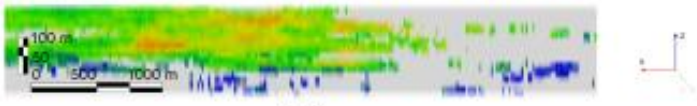

(d)

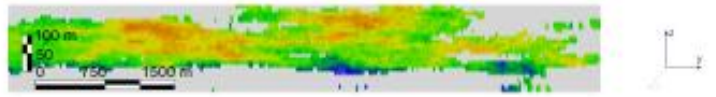

(f)

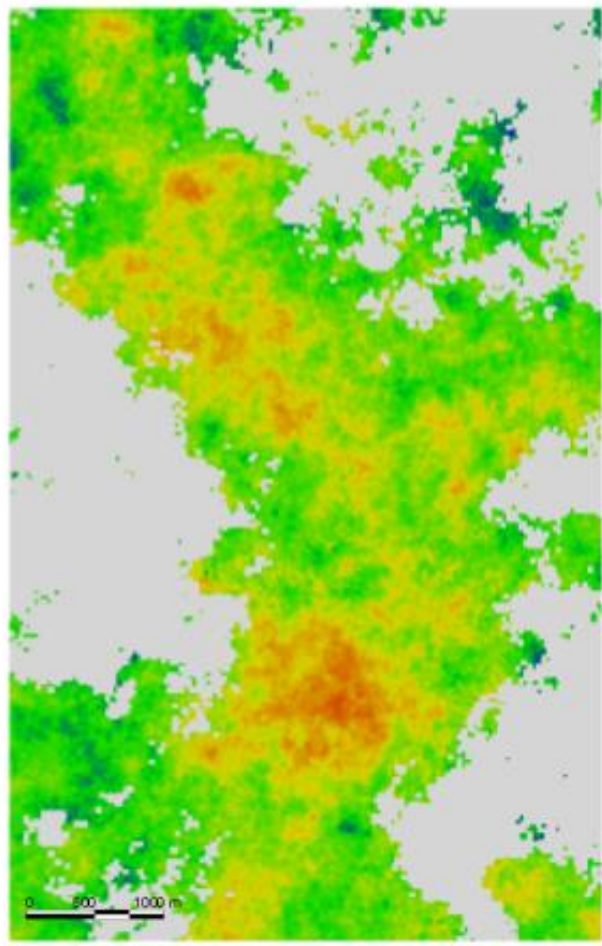

(h)

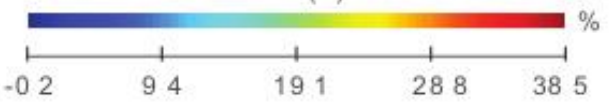

Figura 5 - Mapa E-type da variável DRDN (primeira coluna) e mapa E-type para a porosidade (segunda coluna).As seções horizontais $(g) e(h)$ corresponde a porção inferior do grid tridimensional. A distribuição das litofácies dessas seções sugerem um sistema deposicional de canais confinados alinhados à direção $N$-S, na porção inferior da seção, e um alinhamento na direção NW-SE,na porção superior da seção. Nas seções verticais $(c),(d),(e)$ e $(f)$ é possível observar a superposição dos diversos eventos turbiditícos. 


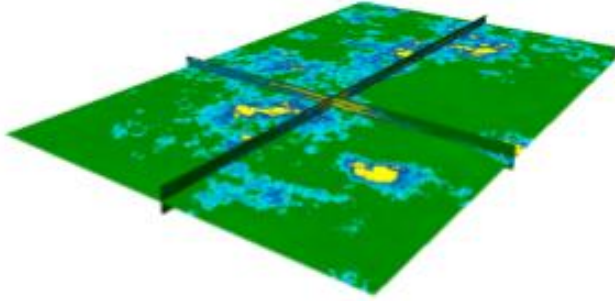

(a)

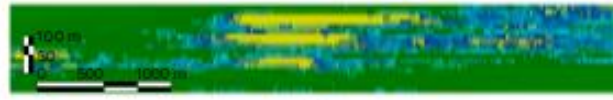

(c)

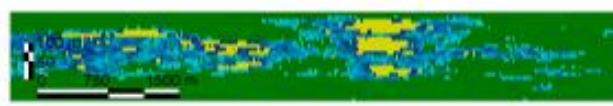

(e)

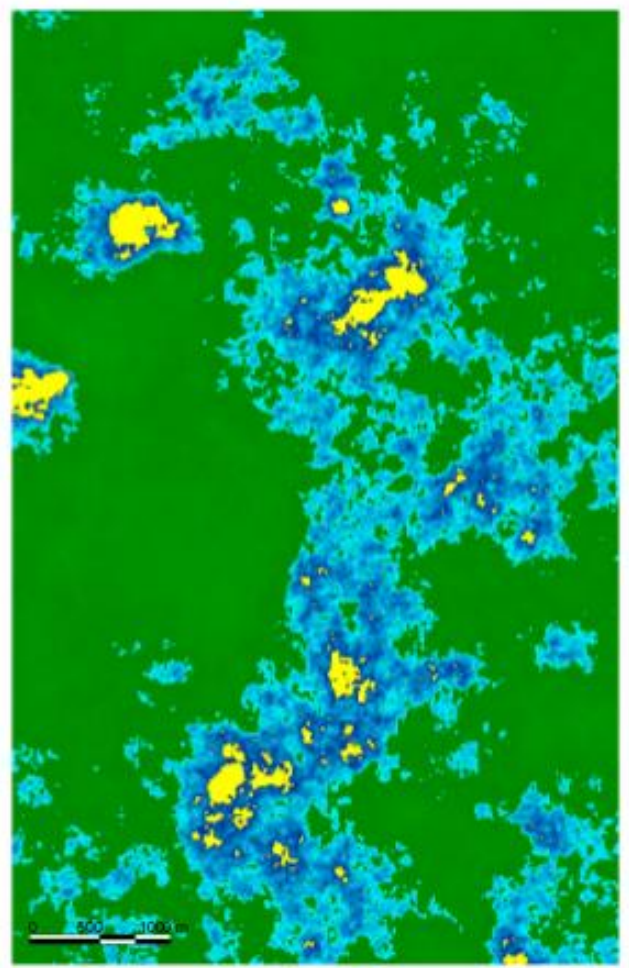

(g)

E NAO RESTRMTORID A RESEIATORIO I RESERMTOROLMPO

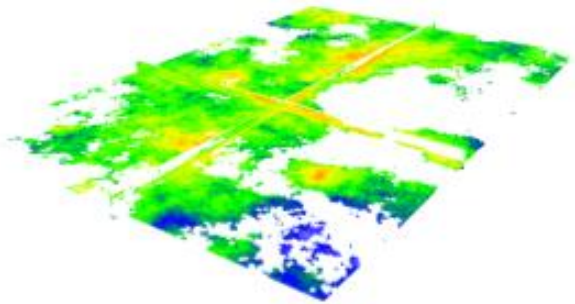

(b)

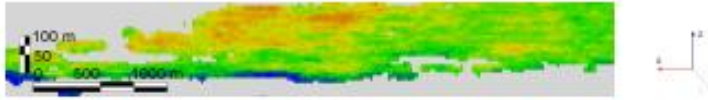

(d)

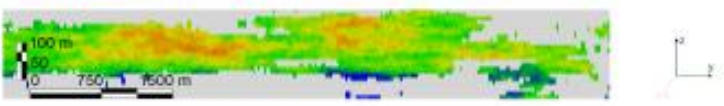

(f)

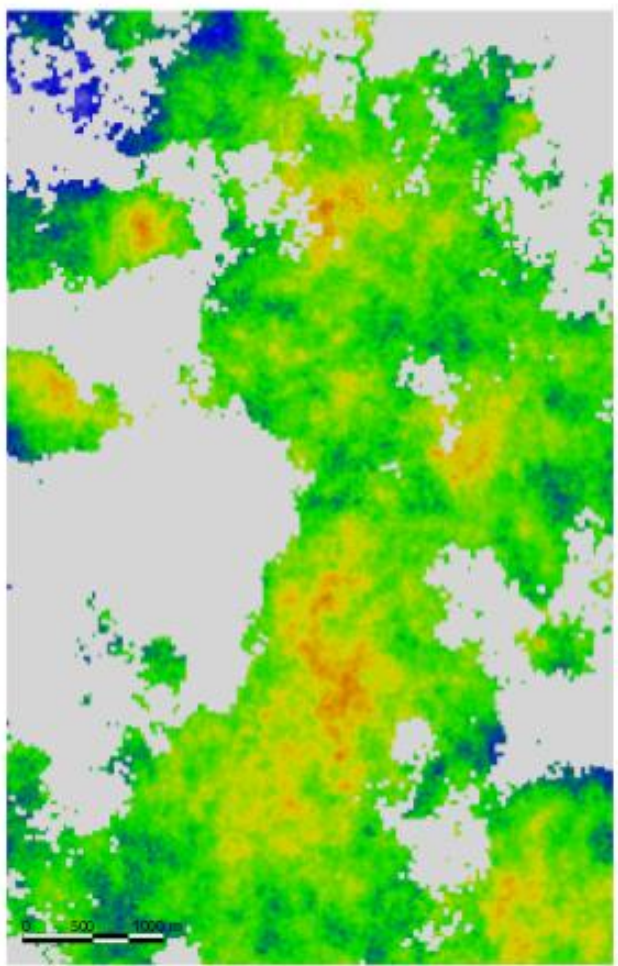

(h)

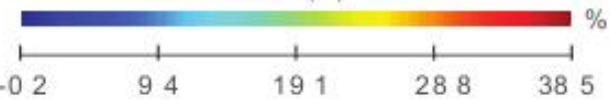

Figura 6 - Mapa E-type da variável DRDN (primeira coluna) e mapa E-type para a porosidade (segunda coluna). As seções horizontais $(g) e(h)$ corresponde a porção superior do grid tridimensional. É possível notar a mudança da direção preferencial dos canais em relação a figura 5. A distribuição das litofácies tornam-se mais espraiadas sugerindo a evolução do sistema deposicional. As seções verticais (c), (d), (e) e (f) mostram os diversos eventos turbiditícos. 


\section{Discussão e Conclusões}

Os métodos geoestatísticos como ferramenta para modelagem de reservatórios turbidíticos, como é o caso do reservatório Namorado, se mostraram bastante eficientes. A simulação sequencial gaussiana foi capaz de representar bem a variabilidade do sistema, dessa forma estando de acordo com as geometrias de deposição propostas tanto por Souza (1997) como por Barboza (2005) para o Campo de Namorado.

Os resultados da simulação sequencial gaussiana, mesmo com as transformações dos poços direcionais em poços verticais, supondo que as medições dos perfis permaneçam na mesma camada estratigráfica, e a translação vertical de todos os poços ao mesmo nível de base do reservatório, apesar de não honrar intencionalmente a posição estrutural real das camadas, mostram que tais transformações honram o modelo deposicional de camadas plano paralelas do reservatório Namorado. Para os objetivos propostos neste estudo, não foi executada a restituição do reservatório para sua posição estrutural real.

A escolha da variável DRDN para a classificação das litofácies foi fundamental para a construção do modelo de reservatório, permitindo mapear o comportamento da distribuição espacial das fácies no reservatório de uma forma mais confiável. Uma vez que o perfil DRDN é uma variável contínua, não sofre perda de informação por truncamento quando transformada em variável categórica, dessa maneira proporcionando mais confiabilidade ao modelo de reservatório obtido.

É importante ressaltar que os depósitos turbidíticos do Campo de Namorado contribuíram para eficácia do método abordado nesse trabalho. A variação lateral esperada para tais depósitos foi capturada pela simulação mesmo com a escassez de dados. A distribuição espacial das fácies simuladas fazem sentido, do ponto de vista do modelo geológico do reservatório, sem impor condições de distribuição espacial além do modelo de variograma obtido através dos dados.

Mesmo com a pobreza de dados e dispondo somente das informações sísmicas para a marcação da base e topo do reservatório, a simulação do reservatório foi capaz de capturar os diferentes eventos turbiditícos como mostram as Figuras 5 e 6 . Isso sugere que 0 modelo poderia ser mais refinado, oferecendo uma maior confiabilidade, se adicionada uma condicionante externa ao conjunto de dados utilizados para a simulação.

Os resultados da validação cruzada mostram que o método apresenta estabilidade mesmo com redução dos dados, dessa forma fornecendo confiabilidade à modelagem do reservatório. Assim, a metodologia apresentada nesse trabalho pode ser usada em cenários exploratórios ou explotatórios onde há pouca disponibilidade de dados sísmicos, como os campos onshore onde a sísmica, em geral, é de baixa qualidade se comparada com as aquisições offshore, e o número de poços é maior devido ao baixo custo relativo às campanhas marítimas.

\section{Referências}

BACOCCOLI, G.; MORALES, R. G.; CAMPOS, O. A. J. The namorado oil field: $A$ major oil discovery in the campos basin, brazil. In:. [S.I.]: American Association of Petroleum Geologists, 1980. cap. Giant Oil and Gas Fields of the Decade: 1968 a 1978.

BARBOZA, E. G. Análise Estratigráfica do Campo Namorado (Bacia de Campos) com base na interpretação Sísmica Tridimensional. Tese (Doutorado) Universidade Federal do Rio Grande do Sul, Porto Alegre, 2005.

BARBOZA, E. G. et al. Análise faciológica e modelo deposicional do arenito namorado, bacia de campos, rj. CONGRESSO BRASILEIRO DE PeD EM PETRÓLEO E GÁS, 2005.

BRUHN, C.H. et al. Campos basin: Reservoir characterization and management historical overview and future challenges. Petrobras EeP, 2003.

CHILES, J.-P.; DELFINER, P. Geostatistics Modeling Spatial Uncertainty. [S.I.]: JOHN WILEY SONS, 1999.

CRUZ, M. M. da. Aplicação de perfilagem geofísica e sísmica na caracterização da faciologia do reservatório Namorado. Dissertação (Mestrado) - Universidade Federal Fluminense, 2003.

DEUTSCH, C. V. Geostatistical Reservoir Modeling. [S.I.]: Oxford University, 2002.

DEUTSCH, C. V.; JOURNEL, A. G. GSLIB Geostatistical Software Library and User's Guide. [S.I.]: Oxford University, 1998.

DIAS, J. L. et al. Aspectos da evolução tectonosedimentar e a ocorrência de hidrocarbonetos na bacia de campos. In: Origem e Evolução de Bacias Sedimentares. 2. ed. Rio de Janeiro: Petrobrás, 1990. cap. 14, p. 333-360.

GUIMARÃES, M. da S. B.; DENICOL, P. S.; GOMES, R. M. R. Avaliação e caracterização de reservatórios laminados: comparação entre as ferramentas convencioais e perfil de indução multicomponente. Revista Brasileira de Geociências, 2008.

ISAAKS, E. H.; SRIVASTAVA, R. M. Introduction an Applied Geostatistics. [S.I.]: OXFORD UNIVERSITY, 1989.

REMACRE, A. Z. Introdução as Funções Aleatórias. 1999.

REMY, N.; BOUCHER, A.; WU, J. Applied Geostatistics with SGeMS. [S.I.]: Cambridge University, 2009.

WEBSTER, R.; OLIVER, M. A. Geostatistics for Environmental Scientists. [S.I.]: JOHN WILEY SONS, 2007.

YAMAMOTO, J. K. Curso de geoestatística aplicada. Periódico Desconhecido, 2001. Notas de aula. 